\title{
Pathogenic aquaporin-4 reactive T cells are sufficient to induce mouse model of neuromyelitis optica
}

\author{
Melina V. Jones, Hwa Huang, Peter A. Calabresi and Michael Levy*
}

\begin{abstract}
Introduction: Neuromyelitis Optica (NMO) is an autoimmune disease primarily targeting the spinal cord and optic nerve leading to paralysis and blindness. The discovery of an antibody against the astrocytic water channel, aquaporin-4 (AQP4), in the majority of patients, has led to the presumption that the antibody was necessary for disease pathogenesis. The potential role of T cells in the central nervous system, however, has not been thoroughly examined.

Results: We generated an anti-AQP4 antibody seronegative model of NMO using pathogenic AQP4-reactive T cells in mice by immunizing AQP4 null mice with peptides corresponding to the second extracellular loop of AQP4, loop C. When polarized to a Th17 phenotype and transferred to wild-type mice, these cells caused tail and limb weakness. Histology showed demyelination and T cell infiltration in the spinal cord, optic nerve and brain. Animals receiving cells re-stimulated in culture with non-specific proteins resulted in no behavioral disease, indicating that specific targeting of AQP4 is essential for this phenotype.
\end{abstract}

Conclusions: In summary, we show that AQP4-reactive T cells are sufficient to trigger an NMO-like disease in mice, independent of antibodies, indicating that pathogenic AQP4-reactive T cells may play a similar role in humans.

Keywords: Neuromyelitis optica, Aquaporin-4, Autoreactive T cells, Th17

\section{Introduction}

Neuromyelitis optica (NMO) is a relapsing autoimmune disease primarily targeting the spinal cord and optic nerve leading to paralysis and blindness [1]. The discovery of the highly specific anti-aquaporin-4 (AQP4) $\mathrm{IgG}_{1}$ biomarker implicates an immune reaction against AQP4 evidenced by both humoral and cellular pathology within acute NMO lesions [2, 3]. Several previous mouse and rat models of NMO focused on the role of the circulating anti-AQP4 antibody in disease pathogenesis concluded that the antibody by itself is insufficient to induce disease, but can exacerbate an experimental autoimmune encephalomyelitis induced by myelin-reactive T cells [4-8]. When the anti-AQP4 antibody has passive access to AQP4 on astrocytes in the nervous system, there is abundant evidence that the antibody binds to AQP4 and can

\footnotetext{
* Correspondence: mlevy@jhmi.edu

Department of Neurology, Johns Hopkins University, 600 N. Wolfe Street, Pathology 509, Baltimore, MD 21287, USA
}

participate in complement mediated damage to astrocytes under experimental conditions [9-11]. Collectively, these studies indicate an important role for the anti-AQP4 antibody in enhancing astrocytic damage from NMO relapses rather than in the triggering of NMO attacks, prompting the search for other AQP4-specific immune components that may be involved upstream in the immunopathogenesis of NMO. In each passive transfer study, the antiAQP4 antibody was only pathogenic in the context of a Tcell based autoimmune attack on the central nervous system.

Production of the $\mathrm{IgG}_{1}$ biomarker against AQP4 presumably requires AQP4-reactive $B$ and $T$ cells for immunoglobulin class switching. $T$ cells are also among the inflammatory cells found in acute NMO lesions and their role in the immunopathogenesis of this disease has been the subject of recent studies in which immunodominant AQP4 peptides can trigger $\mathrm{T}$ cell activation in mice $[12,13]$. However, despite activating $T$ cells against AQP4, these rat and mouse models do not develop a 
clinical neurological phenotype either because pathogenic $\mathrm{T}$ cells responses are limited by a combination of central and peripheral tolerance or because certain AQP4 epitopes are not pathogenic.

We used a unique approach to raise pathogenic AQP4-reactive $T$ cells by immunizing AQP4 null mice with loop C peptide of AQP4 (AQP4 $4_{135-153}$ ). When adoptively transferred into wild type mice after re-stimulation in culture with Aqp4 $4_{135-153}$ peptide, behavioral disease was not induced though mild $\mathrm{T}$ cell infiltration of the central nervous system (CNS) was observed. Polarization of AQP4-reactive $\mathrm{T}$ cells to the T-helper-17 led to CNS inflammation characterized by demyelination and $\mathrm{T}$ cell infiltration into the spinal cord, optic nerve, and brain. There was no other evidence of solid organ inflammation despite widespread AQP4 expression in the mouse supporting the specificity of this approach to modeling the human NMO disease.

\section{Materials and methods Animals}

Aquaporin-4 null mice, backcrossed onto the C57BL/6 background at least 14 times, were obtained from Erlend Nagelhus (University of Oslo, Oslo, Norway) and bred inhouse. Female C57BL/ 6 wild-type mice between $6-8$ weeks of age were purchased from The Jackson Laboratory. All mice were housed in a pathogen-free 12-h artificial lightdark cycle and had ad libitum access to food and water. The Johns Hopkins Institutional Animal Care and Use Committee approved all experimental procedures.

\section{T cell generation and culturing}

Aquaporin-4 extracellular loop peptides (human 56-69, 135-53, and 212-30) were synthesized at the Johns Hopkins Synthesis \& Sequencing Core Facility. Stock solutions of $120 \mathrm{mg} / \mathrm{ml}$ were prepared in DMSO. All three peptides were further diluted into phosphate-buffered saline each at $2 \mathrm{mg} / \mathrm{ml}$ and mixed 1:1 with complete adjuvant containing $8 \mathrm{mg} / \mathrm{ml}$ heat-killed $M$. tuberculosis H37Ra (Difco) in incomplete adjuvant (Imject; Thermo-Fisher) [14]. Aquaporin-4 $\mathrm{KO}$ and syngeneic $\mathrm{C} 57 \mathrm{Bl} / 6$ mice (Jackson, MA, USA) were immunized in the flanks with a total of $100 \mu \mathrm{l}$ of emulsion. Mice were also injected intraperitoneally with $250 \mathrm{ng}$ of Pertussis toxin (Tocris) on days 0 and 2. Twenty-three days following immunization, spleens were harvested and single cell suspensions were prepared by pushing spleens through $70 \mu \mathrm{m}$ cell strainers (Becton Dickenson) using syringe plungers. Red blood cells were depleted by resuspending each spleen in $2 \mathrm{ml}$ of ACK lysis solution (Quality Biological, MD, USA) for $2 \mathrm{~min}$ at room temperature, followed by washing with media. Cells were counted and seeded in 96-well flat bottom plates at $3 \times 10^{5}$ cells per well in RPMI 1640 supplemented with Glutamax, $1 \%$ non-essential amino acids, $1 \%$ sodium pyruvate, $1 \%$ antibiotic-antimycotic (Life Technologies, Inc.), $10 \%$ fetal calf serum (Sigma) and $50 \mu \mathrm{M}$ betamercaptoethanol (Sigma). One hundred microliters of media containing peptide (final concentration: $10 \mu \mathrm{g} /$ $\mathrm{ml}$ ): $\mathrm{MOG}_{35-55}, \mathrm{AQP}_{56-69}$ (loop A), AQP4 $4_{135-53}$ (loop C), or AQP4 $4_{212-30}$ (loop E) were added to wells in triplicate. Media with no peptide added or containing $0.1 \%$ DMSO served as "no stimulus (NS)" background controls. After 4 days in culture in an incubator at $37{ }^{\circ} \mathrm{C}$ with $5 \%$ $\mathrm{CO}_{2}$ in a humidified atmosphere, $10 \mu \mathrm{l}$ of a solution containing $0.5 \mu \mathrm{Cu}$ of ${ }^{3} \mathrm{H}$-thymidine (Perkin-elmer) were added to each well and incubated a further $18 \mathrm{~h}$. Cells were harvested onto filter paper mat. After drying, mats were treated with scintillation fluid and assayed for ${ }^{3} \mathrm{H}$ incorporation. Results are expressed as counts per minute $(\mathrm{cpm})$.

\section{T cell polarization, adoptive transfer and behavioral scoring}

Six to seven week old female aquaporin- $4 \mathrm{KO}$ mice were immunized with an emulsion of $4 \mathrm{mg} / \mathrm{ml}$ Complete Freund's adjuvant with $1 \mathrm{mg} / \mathrm{ml}$ of Loop C peptide (135-53) on each flank and each shoulder $(50 \mu \mathrm{l}$ per injection site). Ten days following immunization, lymph nodes (inguinal, lumbar, brachial and axillary) were collected. Single cell suspensions were prepared by forcing nodes through a $70 \mu \mathrm{m}$ cell strainer using a syringe plunger. After washing in complete media (RPMI 1640 with 10\%FCS, $50 \mu \mathrm{M} \beta$-mercaptoethanol, non-essential amino acids, sodium pyruvate, HEPES), cells were counted and seeded into T75 flasks at $6 \times 10^{6}$ cells per ml. Unpolarized cells were stimulated with peptide only (final concentration was $50 \mu \mathrm{g} / \mathrm{ml}$ ). For Th17 polarization, cells were stimulated with $30 \mathrm{ng} / \mathrm{ml} \mathrm{IL-6,} 20 \mathrm{ng} / \mathrm{ml} \mathrm{IL-23} \mathrm{(eBiosciences)}$ and $10 \mu \mathrm{g} / \mathrm{ml}$ of anti-IFNgamma (XMG1.2; BD Biosciences) in addition to peptide. Following 3 days of culture, cells were harvested, washed with sterile PBS, and $5 \times 10^{6}$ cells were injected into mice intravenously through their tail veins. Pertussis toxin (250 ng; Tocris) was injected intravenously immediately following cells and again two days later. Behavioral signs and weights were tracked starting 5 days post-transfer of cells, which was quantified using a standardized 5-point EAE disability scale by a blinded examiner [14]. Animals were euthanized and tissues harvested for histological evaluation 14-21 days following cell transfer.

\section{ELISPOT assay}

ELISPOT assay was used to determine the frequency of cytokine-producing cells in polarized and unpolarized cell cultures. The day before cells were to be harvested from immunized AQP4 null mice, the wells of an immobilon Pbottom 96-well plates (Multiscreen ${ }^{\circ} \mathrm{HTS}, 0.45 \mu \mathrm{m}$ pore size; EMB Millipore, USA) were pre-wet with $35 \%$ ethanol 
for $30 \mathrm{~s}$, washed three times with coating buffer, and coated with $50 \mu \mathrm{l}$ of a 1:250 dilution of capture antibody, anti-IL17 (Th17), provided with ELISPOT Ready-Set-Go kits (eBioscience). Plates were covered and incubated overnight at $4{ }^{\circ} \mathrm{C}$. Wells were then washed twice with coating buffer and once with complete media; plates were stored in the incubator until cells were prepared. Spleen and lymph node cells were prepared as described above and prepared with and without polarizing conditions. Harvested cells were 2 -fold serially diluted in media containing $3 \times 10^{7}$ irradiated splenocytes (irradiated with 3,500 rads) as antigen-presenting cells (APCs); APCs mixed 12:1 with $1.25 \times 10^{5}$ lymph node cells per well yielded the most welldefined spots. After overnight in culture, wells were washed (TBS + $0.05 \%$ Tween $\left.^{\circ}-20\right)$. Detection antibody was diluted 1:250 in diluents provided in the kit and $50 \mu \mathrm{l}$ applied to each well for $2 \mathrm{~h}$ at RT. After washing, streptavidin-alkaline phosphatase was added to each well (1:2500; Sigma) for $45 \mathrm{~min}$. After further washing, signal was developed with development solution containing BCIP and NBT (i.e. $150 \mathrm{mM}$ Tris-HCl, $5 \mathrm{mM} \mathrm{MgCl}{ }^{2}, 100 \mathrm{mM} \mathrm{NaCl}$, pH9.5 supplemented with $4 \mathrm{mM}$ levamisole (Sigma), $0.15 \mathrm{mg} / \mathrm{ml}$ 5-bromo-4-chloro-3-indolyl-phosphate, and $0.36 \mathrm{mg} / \mathrm{ml} \mathrm{4-}$ nitro blue tetrazolium chloride (Roche) for $10 \mathrm{~min}$ at room temperature in the dark. Reaction was stopped by washing with $\mathrm{PBS}$ then distilled $\mathrm{H}^{2} \mathrm{O}$ before air drying. Spots were imaged with an Immunospot Series 3 Analyzer (Cellular Technology, Ltd.) and counted using Image J and the "find Maxima" function. Results are expressed as the mean of triplicate values $( \pm$ standard error of the mean, SEM) adjusted to per $10^{6}$ cells per well. Student's $t$-test was performed on data; $\mathrm{p}<0.05$ was considered statistically significant.

\section{Tissue processing and histology}

Animals were anesthetized with isofluorane and perfused via cardiac puncture first with PBS and then with freshly prepared $4 \%$ paraformaldehyde solution. The optic nerves and spinal cords were harvested, fixed overnight, cryopreserved in $30 \%$ sucrose and frozen for sectioning. After embedding tissue in O.C.T. Compound (Tissue-Tek ${ }^{\circ}$ ), ten micron slices sections were mounted on Superfrost Plus Microscope Slides (Fisher brand). Eriochrome cyanine was used to identify demyelinating lesions in the sectioned tissue. Eriochrome cyanine solution was prepared by dissolving eriochrome cyanine in $450 \mathrm{ml} 0.5 \% \mathrm{H}_{2} \mathrm{SO}_{4}(0.2 \%)$ and $10 \% \mathrm{FeCl}_{3}$ added to a final concentration of $0.4 \%$. The sectioned tissue was hydrated by serial washes in $100 \%$ ethanol, $95 \%$ ethanol, $70 \%$ ethanol and distilled water for $10 \mathrm{~min}$ each and then immersed for $15 \mathrm{~min}$ in eriochrome cyanine solution. After staining, differentiation was carried out in freshly made $0.1 \% \mathrm{NH}_{4} \mathrm{OH}$ for $20-30 \mathrm{~s}$ and halted by thorough washing in distilled water. Slides were mounted as described below. Sections were counterstained with $0.1 \%$ eosin $\mathrm{Y}$ in acetate buffer. Immunohistochemical staining for $\mathrm{CD} 3+\mathrm{T}$ cells was performed by washing sections in saline before performing heat-mediated antigen retrieval in $0.05 \mathrm{M}$ sodium borate buffer $(\mathrm{pH}$ 8.0) in a microwave pressure cooker. Slides immersed in buffer were heated in the microwave at full power until full pressure was achieved $(5 \mathrm{~min}$ ) then heated for an additional $7 \mathrm{~min}$ at $20 \%$ power. After 3 min of cooling and flooding of slide container with room temperature saline, slides were transferred to $3 \% \mathrm{H}_{2} \mathrm{O}_{2}$ for $20 \mathrm{~min}$ to quench endogenous peroxidases and blocked for endogenous biotin using an avidin/biotin blocking kit (Vector Laboratories, Inc.). Non-specific binding was blocked with $5 \%$ goat serum in $0.1 \%$ Triton $^{\circ} \mathrm{X}-100$ for $30 \mathrm{~min}$ at room temperature. Anti-CD3 rabbit monoclonal antibody (clone SP7; GeneTex,USA) was applied at 1:75 overnight at $4{ }^{\circ} \mathrm{C}$ and detected with biotinylated goat antirabbit IgG (1:1000; Vector Laboratories, Inc.), followed by Avidin-Biotin Complex-horse radish peroxidase (Vector Laboratories, Inc.). Signal was developed with $0.5 \mathrm{mg} / \mathrm{ml}$ diaminobenzidine $\mathrm{HCl}$ in PBS with $0.03 \% \mathrm{H}_{2} \mathrm{O}_{2}$ for $5 \mathrm{~min}$. After washing, slides were Fast Green counterstained, dehydrated and mounted. Quantification and analysis of myelin and CD3 staining was as described [7]. Glial fibrillary acidic protein (GFAP), myelin basic protein for myelin, and aquaporin-4 were examined by immunofluorescence (without antigen retrieval) applying mouse anti-GFAP (1:1000; Sigma), rabbit monoclonal anti-MBP (1:250; Epitomics/ Abcam), and rabbit anti-AQP4 (H-19) (1:250; Santa Cruz Biotechnology) overnight at $4{ }^{\circ} \mathrm{C}$, followed by goat Alexa Fluor ${ }^{\circ}$ 555-conjugated anti-rabbit IgG and Alexa Fluor ${ }^{\circ}$ 488-conjugated anti-mouse IgG (1:250; Life Technologies / Molecular Probes) for $30 \mathrm{~min}$ at RT. Fluorescent sections were mounted with Fluorogel (Electron Microscopy Sciences) containing $2 \mu \mathrm{g} / \mathrm{ml} \mathrm{4}$, 6Diamidino-2-Phenylindole (DAPI) and sealed with clear nail polish.

CD3 quantification was performed in a blinded fashion using background-corrected high resolution images from areas representing 6-8 levels of the spinal cord, 3 coronal levels of the cerebrum (left and right, for a total of 6 fields of view captured at $4 \mathrm{X}$ ), or a region from the left and right lobes of the cerebellum (2 fields at $4 \mathrm{X}$ ) via Fast Green staining. Three sections from each optic nerve were also analyzed (each representing a part of the longitudinal length of the nerves). Total immunoreactive (brown DAB reaction product) was expressed as \% immunoreactive area and mean \pm SEM.

\section{Results}

In NMO, the anti-AQP4 antibody targets an extracellular epitope of AQP4 [15]. Using AQP4 null mice, we checked for antibody production and $\mathrm{T}$ cell responses against peptides corresponding to the extracellular loops of AQP4, 
loops A, C and E. AQP4 null mice were used in this model because these mice do not have to overcome immune tolerance to develop pathogenic AQP4-reactive T cells. We found no evidence of antibody production against any of the loops. The lack of anti-AQP4 antibody production is not unexpected as short peptides do not routinely exhibit robust antibody responses. However, there was a robust $\mathrm{T}$ cell response against the second extracellular loop of AQP4, loop C (Fig. 1a). In phenotyping these AQP4-reactive $T$ cells, we found that a significant number of these unpolarized cells produced both interleukin-17 (IL17) and IFN-gamma, compared to non-stimulus (NS) controls (Fig. 1b). Polarization of $\mathrm{T}$ cells towards the Th17 phenotype (Th17-pol) produced twice the number of IL17-secreting cells while the number of IFN-gamma producing cells were barely detectable (Fig. 1b).

The AQP4 null mice that generated a robust $\mathrm{T}$ cell reaction against the second extracellular loop of AQP4, loop $\mathrm{C}$, did not develop autoimmune neurological disease as they do not express the target antigen. Even when intravenously transferred to wildtype AQP4-expressing C57Bl/
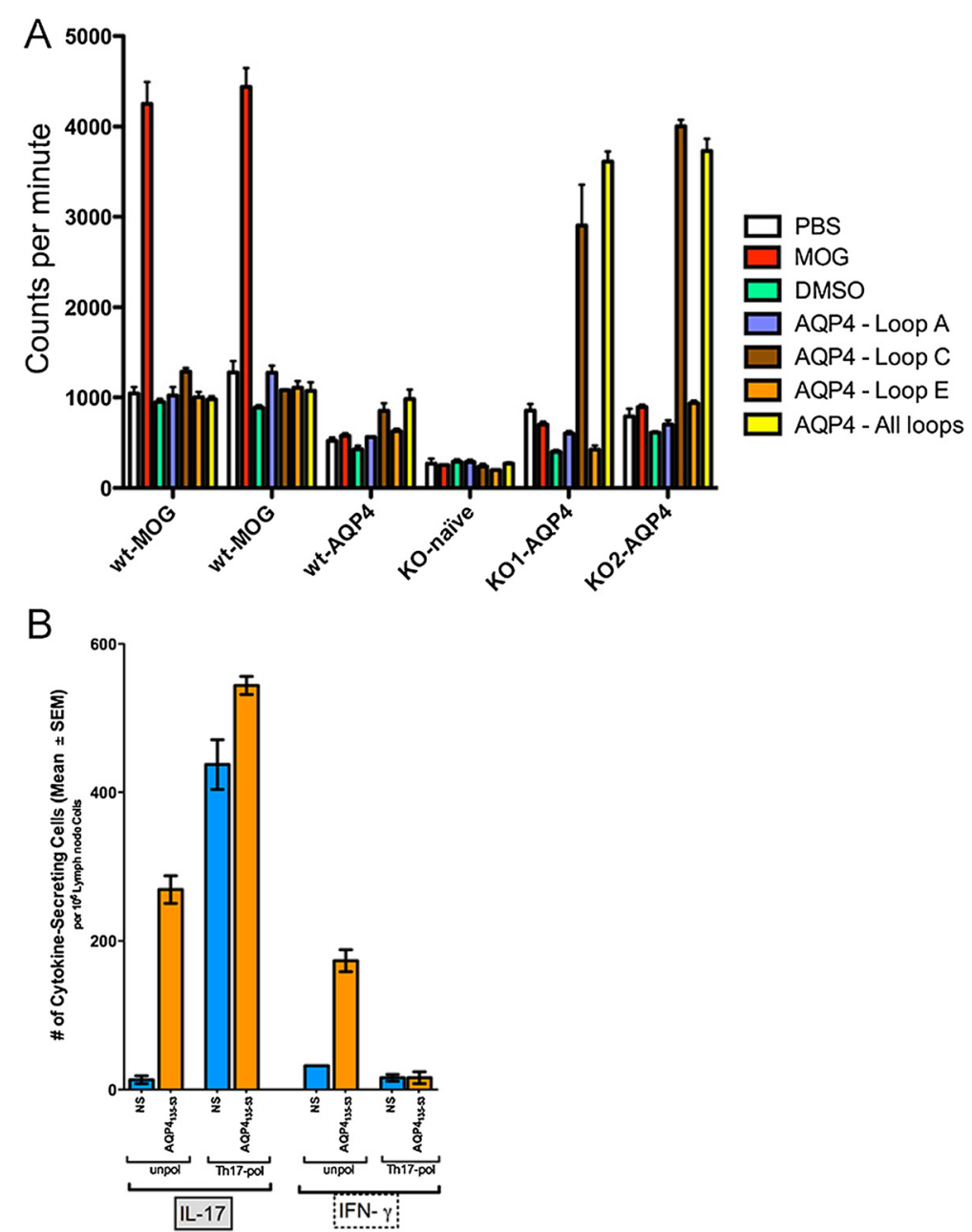

Fig. 1 A. T cell proliferation assay reflecting a robust reaction to loop C of AQP4. T cells from two C57Bl6 mice (wt-MOG) immunized with myelin-oligodendrocyte glycoprotein (MOG) peptide 35-55 showed proliferative activity as measured by incorporation of tritiated-thymidine only in the presence of the MOG antigen (red bars). C57Bl6 mice immunized with AQP4 peptides (wt-AQP4) did not react against any AQP4 antigen. T cells from AQP4 null mice (KO naïve) do not inherently react to AQP4 peptides unless the mice are immunized with the peptides (KO1-AQP4 and KO2-AQP4). Among those tested, a peptide corresponding to the extracellular loop C generated a robust reaction only in T cells from AQP4 null mice immunized against a mix of AQP4 peptides 56-69 (loop A), 135-53 (loop C), and 212-30 (loop E), exposed to cells in culture alone (brown bars) or as a mixture of all three peptide (yellow bars). Results shown are counts per minute means +/- SEM of triplicate reactions. B. ELISPOT assay was used to determine the number of IL-17 and interferon-gamma (IFN- - ) cytokine-producing cells in Th17 polarized (Th17-pol) and unpolarized (unpol) cell cultures exposed to AQP4 loop C peptide (AQP4 135-53) versus no stimulation (NS). Unpolarized AQP4-reactive T cells expressed significant levels of both IL-17 and IFN- $\gamma$ compared to unstimulated controls. After polarization to a Th17 phenotype, the number of IL-17 producing cells almost doubles while the number of IFN- $\gamma$ producing cells is nearly undetectable, but the frequency of IL-17-producing cells also increases in the unstimulated culture (while IFN- - -producing cells remains low) 
6 mice, these AQP4-reactive T cells did not develop clinically meaningful behavioral manifestations or histological evidence of CNS demyelinating disease beyond meningeal inflammation (data not shown). Prior to adoptive transfer, when AQP4-reactive $\mathrm{T}$ cells were polarized to a stronger pro-inflammatory Th17 helper cell phenotype, the clinical effect was dramatic with leg weakness and paralysis in addition to a drooping tail with behavioral scores of at least 2.0 (Fig. 2) and associated weight loss.

Three important controls showed no clinical or histological phenotype, confirming the specificity of this model. Adoptive transfer of Th17 polarized AQP4reactive $\mathrm{T}$ cells from a. unstimulated cultures or b. cultures stimulated with non-specific proteins was harmless highlighting the requirement of AQP4peptide during the polarization process (Fig. 2). Pathogenic AQP4-reactive $\mathrm{T}$ cells polarized to Th17 that were transferred back to naïve AQP4 null mice is also harmless demonstrating that astrocytic expression of AQP4 in the host mouse is necessary for this model (data not shown).

Histologically, in clinically asymptomatic wildtype recipients of unpolarized AQP4-reactive T cells, there were rare $\mathrm{CD} 3+\mathrm{T}$ cells scattered in the parenchyma of the spinal cord, optic nerve and brain (Fig. 3a, d, g), as well as other AQP4-expressing solid organs such as the lung (Fig. 3j). In clinically-affected wildtype recipients of adoptively transferred Th17-polarized AQP4-reactive T
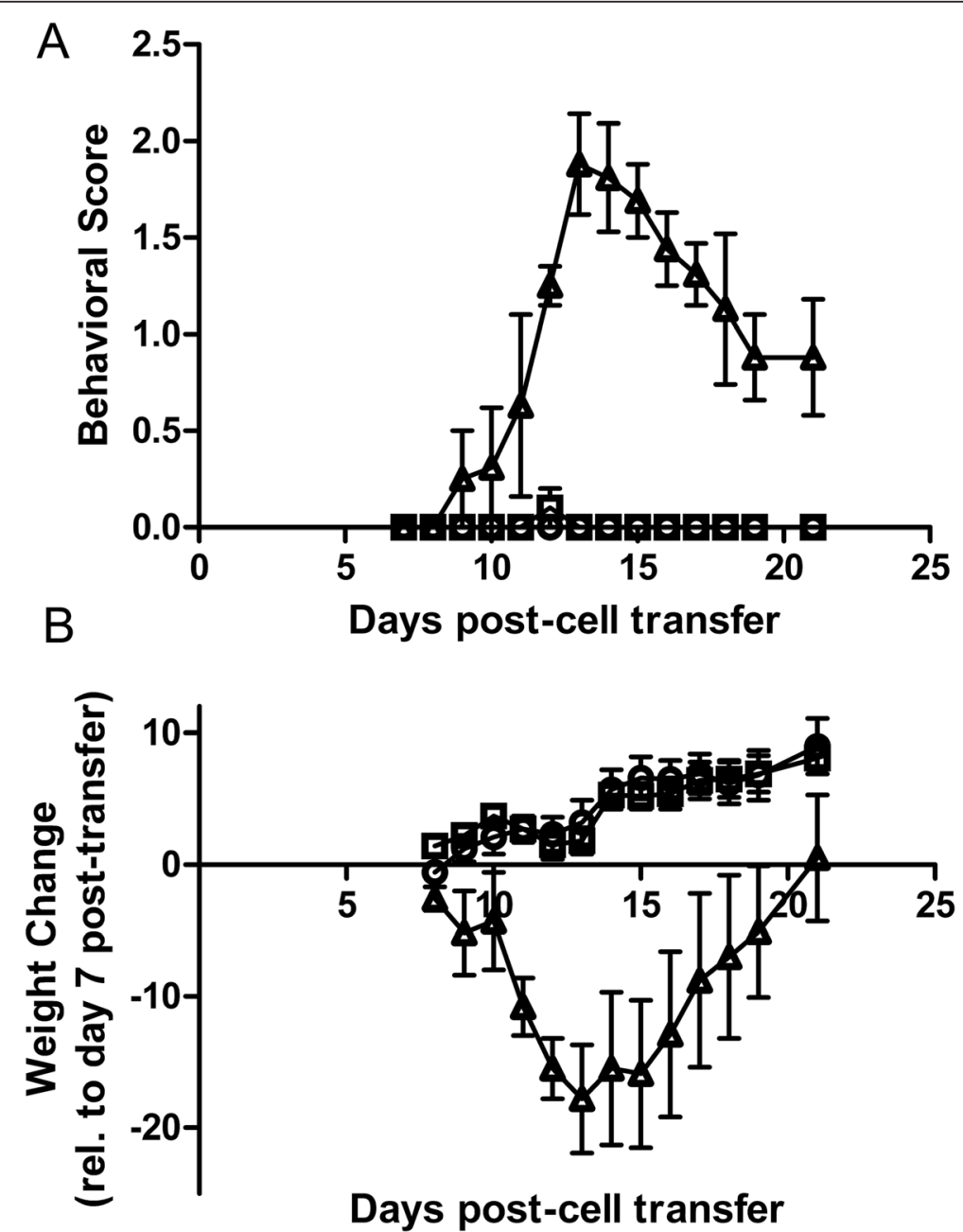

Fig. 2 A. Behavioral assessment of adoptive intravenous transfer of T cells from AQP4 null mice immunized against loop C peptide of AQP4. The Behavior Score is a 5 point EAE-scale scoring the extent of neurologic disability ( 0 is no disability, 5 is death). Wildtype mice (triangles) adoptively transferred with cultured AQP4-restimulated, Th17 polarized AQP4-reactive T cells develop weakness in the tail and hind limbs (EAE score of 1.0-2.0, $n=4$ ). Transfer of AQP4-reactive T cells that are not re-stimulated with AQP4 peptide (squares, $n=5$ ) or stimulated with non-specific proteins (circles, $n=6)$ show no behavioral phenotype. B. Daily weights show typical weight loss in mice receiving the Th17 polarized AQP4-reactive and AQP4-restimulated T cells (triangles), but not in mice that receive unstimulated (circles) or non-AQP4-specifically re-stimulated T cells (squares) 

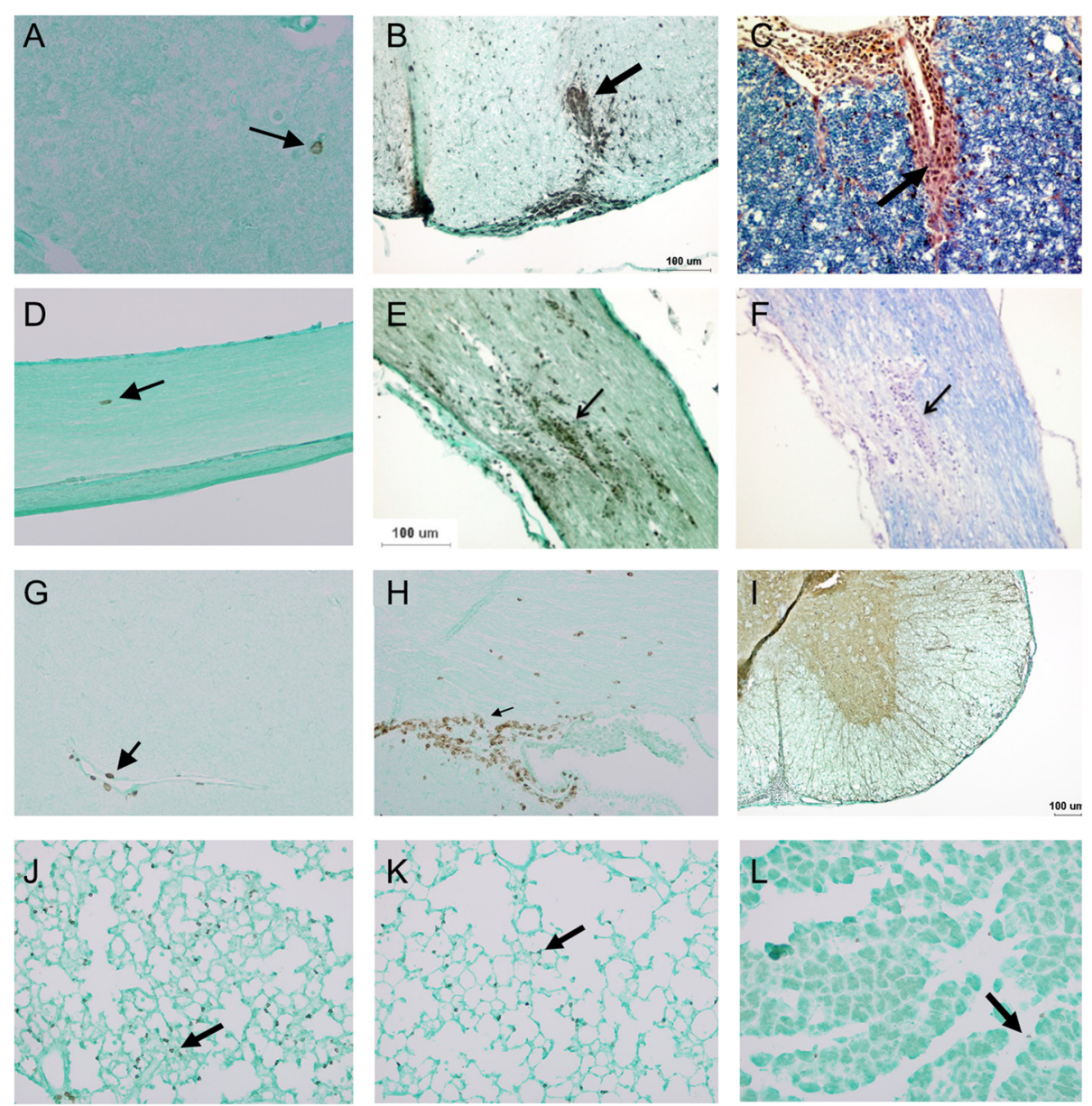

Fig. 3 Histology of tissues from wildtype mice receiving unpolarized AQP4-reactive T cells (A, D, G, J) versus wildtype mice receiving Th17-polarized AQP4-reactive T cells (B, C, E, F, H, I, K, L). A. Spinal cord parenchyma stained for CD3+ T cells shows rare, scattered cell (arrow), compared to B. spinal cord sections from wildtype recipients of Th17-polarized AQP4-reactive T cells which shows intense perivascular CD3+ T cell infiltrates. $\mathbf{C}$. Areas of demyelination (red) within white matter tracts (blue) are visible within inflammatory lesions. D. Longitudinal sections of optic nerves stained for CD3+ T cells shows rare, scattered cell (arrow), compared to $\mathbf{E}$. optic nerve sections from wildtype recipients of Th17-polarized AQP4-reactive T cells which shows intense perivascular CD3+ T cell infiltrates (arrow) F. Areas of demyelination (arrow pointing to red) within white matter tracts (blue) are visible within inflammatory lesions. G. Brain parenchyma stained for CD3+ T cells shows rare, scattered cell (arrow), compared to $\mathbf{H}$. a brain section from wildtype recipients of Th17-polarized AQP4-reactive T cells which shows intense CD3+ T cell infiltrates, such as this lesion around the $3^{\text {rd }}$ ventricle (arrow). I. AQP4-reactive T cells do not appear to change AQP4 staining either in lesions or in normal appearing spinal cord, optic nerve or brain despite widespread inflammation and demyelination (representative section from spinal cord shown). J. Despite expression of AQP4 in solid organs, rare AQP4-reactive CD3+ T cells appear scattered throughout these organs both in the unpolarized and Th17-polarized wildtype recipients. Lung from unpolarized shown here with arrow pointing to CD3+ cells. $\mathbf{K}$. Lung section from Th17-polarized recipient showing normal lung with occasional CD3+ cells (arrow). L. Muscle from Th17 polarized mice show no evidence of inflammation (arrow pointing to rare CD3+ T cells)

cells, histology revealed demyelination and increased inflammatory infiltrate comprised primarily of CD3+ lymphocytes in the spinal cord, optic nerve and brain. Inflammation and demyelination in the spinal cord (Fig. 3b, c) and optic nerves (Fig. 3e, f) accounted for the majority of symptoms, but parts of the brainstem, cerebellum and cerebral cortex showed areas of inflammation that were not as clinically obvious in the mice (Fig. 3h). Although the AQP4 water channel is targeted by these pathogenic $\mathrm{T}$ cells, astrocytic AQP4 expression appears relatively intact even within acute inflammatory lesions (Fig. 3i). Despite AQP4 expression in many other solid organs, there is no evidence of inflammation or AQP4 loss outside of the CNS including the lung (Fig. 3k) or muscles (Fig. 3L) in clinically affected mice. Blinded quantification of CD3 cells in the spinal cord, optic nerve, and brain of wildtype recipients of Th17polarized AQP4-reactive $\mathrm{T}$ cells shows $>5$-fold more immunoreactivity $(* *<0.01)$ compared to mice that received unpolarized AQP4-reactive T cells (Fig. 4). 


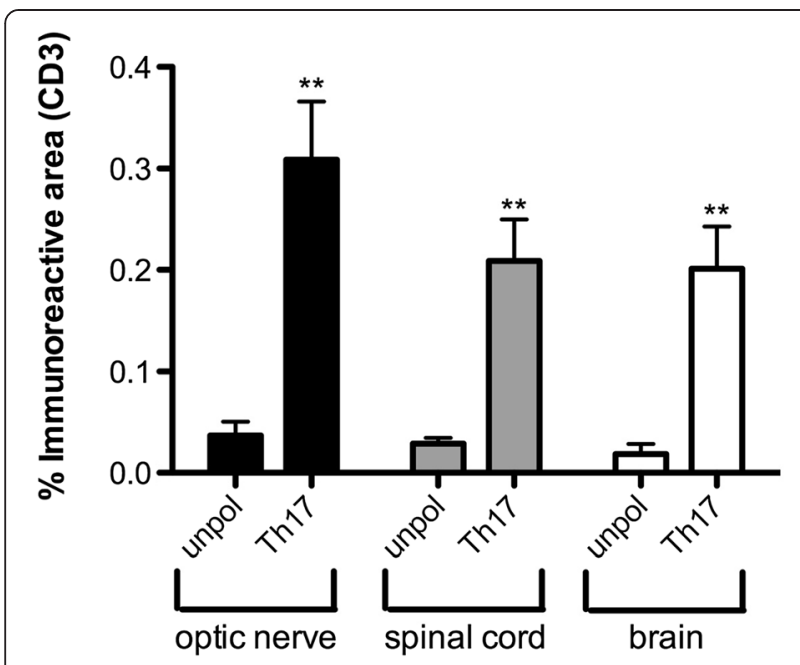

Fig. 4 Blinded quantification of $C D 3$ cells in the spinal cord $(n=8)$, optic nerve $(n=6)$ and brain $(n=8)$ of wildtype recipients of Th17-polarized AQP4-reactive T cells shows $>5$-fold more immunoreactivity $\left({ }^{* *} p<0.01\right)$ in these tissues compared to unpolarized mice

\section{Discussion}

This study demonstrates that adoptive intravenous transfer of pathogenic AQP4-reactive T cells is sufficient to cause an NMO-like inflammatory disease that attacks the optic nerves, spinal cord and brain while sparing other AQP4expressing non-CNS organs. Polarization of AQP4-reactive $\mathrm{T}$ cells to a stronger pro-inflammatory Th17 phenotype prior to transfer amplifies the inflammation leading to more severe demyelination and neurological dysfunction. The best explanation for this response is that immunization with AQP4-loop C peptide in AQP4 null mice generates a $T$ cell response that is different from immunization of the same loop in wildtype mice [16]. The AQP4-reactive T cells in null mice are not exposed to any degree of negative selection as they likely would be if raised in wildtype mice. There may also be a protective regulatory response in wildtype mice that suppresses any potential autoreactive tendency.

The implications of this study point to a key immunopathogenic role of Th17 polarized AQP4-reactive T cells of NMO in both triggering and localizing inflammation to AQP4 within the central nervous system. Pathogenic $\mathrm{T}$ cells targeting AQP4 do not kill AQP4-expressing astrocytes and do not cause loss of AQP4 expression. Rather, their role as demonstrated in this model is to trigger attacks directed towards AQP4-rich areas of the CNS and then recruit other components of the immune system, including antibodies and complement, to mediate the astrocytic damage. In human NMO pathology, death of astrocytes and loss of aquaporin- 4 is a more downstream event initiated with binding of anti-AQP4 antibody, which leads to either complement-mediated destruction of the M23 isoform or internalization of the M1 isoform of AQP4 [17, 18]. We and others have previously demonstrated a pathogenic function of the antiAQP4 antibody in exacerbating neuroinflammation in rodents, but not in instigating the disease [5-7]. The specificity of the immune target to the nervous system is not mediated by the anti-AQP4 antibody as it will bind AQP4 in any organ [19].

Interestingly, the extracellular loop $\mathrm{C}$ is the most common target of the anti-AQP4 antibody in humans [15]. AQP4-reactive T cells and a pathogenic anti-AQP4 antibody may work together to cause NMO. In this model, a susceptible person is exposed to a peptide corresponding to loop C of AQP4 under conditions that stimulate both an auto-reactive $\mathrm{T}$ cell and antibody reaction. A proinflammatory Th17 response to AQP4 may cause a more fulminant disease as demonstrated in this study and previous animal models [20]. Only after AQP4-reactive T cells trigger inflammation directed to the optic nerves and spinal cord would anti-AQP4 antibodies exacerbate the pathology by fueling complement activation and granulocyte recruitment.

This model highlights the potential for AQP4-specific immunotherapy for NMO. As a disease with a highly specific antigen (AQP4) and antibody response (anti-AQP4) associated with AQP4-reactive T cells, NMO is poised for treatment with an antigen-specific therapy [21, 22]. To induce a tolerance response, high dose soluble loop $C$ peptide may be provided to patients in the setting of immunosuppression commonly used to treat NMO currently. With pre-existing disease, an oral route to achieve mucosal tolerance may be the safest initial approach to avoid worsening the disease [23]. In other diseases with less antigen-specificity, such as rheumatoid arthritis and multiple sclerosis, there is significant heterogeneity in the immunodominant antigen responses; in contrast, NMO is defined by reaction primarily to the AQP4 water channel although the precise target within AQP4 may vary slightly among NMO patients with some patients producing antibody responses against loops A and $\mathrm{E}$ as well [19]. A study in Lewis rats in which $\mathrm{T}$ cells reactive against loop E could induce inflammation in the spinal cord suggests that extracellular targets of AQP4 other than loop $\mathrm{C}$ may be involved [24].

\section{Conclusions}

This model demonstrates the potential of AQP4-reactive $\mathrm{T}$ cells to contribute to behavioral disease and to oligodendrocyte damage in the central nervous system, independent of their peripheral role in generating high affinity astrocytoxic antibodies. New therapeutic options targeting such Th17 AQP4-reactive T cell in patients suffering from NMO could be examined in this model. Further study is required to determine how oligodendrocytes are killed by $\mathrm{T}$ cells 
reactive to an astrocyte protein. Indeed, how Th1 and Th17 $\mathrm{T}$ cells indirectly contribute to cellular damage through direct interactions with the antigen-presenting cells remains an unanswered question in NMO and multiple sclerosis pathogenesis. While antibody activation of complement and Fcreceptor-mediated cytotoxicity are well-established as important mediators in NMO pathology, this model provides a tool for examining $\mathrm{T}$ cell-mediated antibody-independent pathways in disease pathogenesis.

\section{Competing interests}

None of the authors have any competing interests to declare.

\section{Authors' contributions}

$M L$ designed experiments, interpreted results and composed the manuscript. MVJ assisted in design of experiments, performed most experiments and assisted with composing the manuscript. Experiments were also performed by HH. PAC assisted with study design and interpretation of results. All authors read and approved the final manuscript.

\section{Acknowledgements/Funding}

This study was funded by a K08 grant from the National Institute of Neurologic Disease and Stroke, grant NS078555 and a grant from the Guthy Jackson Charitable Foundation. We would like to thank Erlend Nagelhus, Ole Ottersen and Peter Agre for providing the AQP4 null mice and Noel Rose for critical input.

\section{Disclosures}

Dr. Levy receives research support from NIH, Guthy Jackson Charitable Foundation, Acorda, Sanofi, NeuralStem and Genentech, and serves as a consultant for Chugai Pharmaceuticals, Glaxo-Smith-Kline, Medlmmune. Dr. Jones and Mr. Huang have no disclosures to report. Dr. Calabresi has received personal compensation for consulting and serving on scientific advisory boards from; Vertex, Vaccinex, Medimmune, Prothena, and Abbott; and has received research funding from companies; Biogen-IDEC and Novartis.

Received: 30 April 2015 Accepted: 30 April 2015

Published online: 21 May 2015

\section{References}

1. Oh J, Levy M (2012) Neuromyelitis optica: an antibody-mediated disorder of the central nervous system. Neurol Res Int 2012:460825

2. Matsuoka T, Suzuki SO, Suenaga T, Iwaki T, Kira J (2011) Reappraisal of aquaporin-4 astrocytopathy in Asian neuromyelitis optica and multiple sclerosis patients. Brain Pathol 21:516-532

3. Popescu BF, Lucchinetti CF (2012) Pathology of demyelinating diseases. Annu Rev Pathol 7:185-217

4. Bennett JL, Lam C, Kalluri SR, Saikali P, Bautista K, Dupree C, Glogowska M, Case D, Antel JP, Owens GP, Gilden D, Nessler S, Stadelmann C, Hemmer B (2009) Intrathecal pathogenic anti-aquaporin-4 antibodies in early neuromyelitis optica. Ann Neurol 66:617-629

5. Bradl M, Misu T, Takahashi T, Watanabe M, Mader S, Reindl M, Adzemovic M, Bauer J, Berger T, Fujihara K, Itoyama Y, Lassmann H (2009) Neuromyelitis optica: pathogenicity of patient immunoglobulin in vivo. Ann Neurol 66:630-643

6. Kinoshita M, Nakatsuji Y, Kimura T, Moriya M, Takata K, Okuno T, Kumanogoh A, Kajiyama K, Yoshikawa H, Sakoda S (2009) Neuromyelitis optica: passive transfer to rats by human immunoglobulin. Biochem Biophys Res Commun 386:623-627

7. Saini H, Rifkin R, Gorelik M, Huang H, Ferguson Z, Jones MV, Levy M (2013) Passively transferred human NMO-lgG exacerbates demyelination in mouse experimental autoimmune encephalomyelitis. BMC Neurol 13:104

8. Ratelade J, Asavapanumas N, Ritchie AM, Wemlinger S, Bennett JL, Verkman AS (2013) Involvement of antibody-dependent cell-mediated cytotoxicity in inflammatory demyelination in a mouse model of neuromyelitis optica. Acta Neuropathol 126:699-709

9. Kinoshita M, Nakatsuji Y, Kimura T, Moriya M, Takata K, Okuno T, Kumanogoh A, Kajiyama K, Yoshikawa H, Sakoda S (2010) Anti-aquaporin-4 antibody induces astrocytic cytotoxicity in the absence of CNS antigenspecific T cells. Biochem Biophys Res Commun 394:205-210

10. Saadoun $S$, Waters $P$, Bell BA, Vincent A, Verkman AS, Papadopoulos MC (2010) Intra-cerebral injection of neuromyelitis optica immunoglobulin G and human complement produces neuromyelitis optica lesions in mice. Brain 133:349-361

11. Saadoun S, Waters P, Macdonald C, Bridges LR, Bell BA, Vincent A, Verkman AS, Papadopoulos MC (2011) T cell deficiency does not reduce lesions in mice produced by intracerebral injection of $\mathrm{NMO}-\mathrm{lgG}$ and complement. J Neuroimmunol 235:27-32

12. Nelson PA, Khodadoust M, Prodhomme T, Spencer C, Patarroyo JC, Varrin-Doyer M, Ho JD, Stroud RM, Zamvil SS (2010) Immunodominant T cell determinants of aquaporin-4, the autoantigen associated with neuromyelitis optica. PLoS One 5, e15050

13. Kalluri SR, Rothhammer V, Staszewski O, Srivastava R, Petermann F, Prinz M, Hemmer B, Korn T (2011) Functional characterization of aquaporin-4 specific T cells: towards a model for neuromyelitis optica. PLoS One 6, e16083

14. Jones MV, Nguyen TT, Deboy CA, Griffin JW, Whartenby KA, Kerr DA, Calabresi PA (2008) Behavioral and pathological outcomes in MOG 35-55 experimental autoimmune encephalomyelitis. J Neuroimmunol 199:83-93

15. Iorio R, Fryer JP, Hinson SR, Fallier-Becker P, Wolburg H, Pittock SJ, Lennon VA (2013) Astrocytic autoantibody of neuromyelitis optica (NMO-lgG) binds to aquaporin-4 extracellular loops, monomers, tetramers and high order arrays. J Autoimmun 40:21-27

16. Lin RH, Stockinger B (1989) T cell immunity or tolerance as a consequence of self antigen presentation. Eur J Immunol 19:105-110

17. Hinson SR, Romero MF, Popescu BF, Lucchinetti CF, Fryer JP, Wolburg H, Fallier-Becker P, Noell S, Lennon VA (2012) Molecular outcomes of neuromyelitis optica (NMO)-lgG binding to aquaporin-4 in astrocytes. Proc Natl Acad Sci U S A 109:1245-1250

18. Rossi A, Ratelade J, Papadopoulos MC, Bennett JL, Verkman AS (2012) Neuromyelitis optica lgG does not alter aquaporin-4 water permeability, plasma membrane M1/M23 isoform content, or supramolecular assembly. Glia 60:2027-2039

19. Saadoun S, Waters P, Leite MI, Bennett JL, Vincent A, Papadopoulos MC (2013) Neuromyelitis optica lgG causes placental inflammation and fetal death. J Immunol 191:2999-3005

20. Herges K, de Jong BA, Kolkowitz I, Dunn C, Mandelbaum G, Ko RM, Maini A, Han MH, Killestein J, Polman C, Goodyear AL, Dunn J, Steinman L, Axtell RC (2012) Protective effect of an elastase inhibitor in a neuromyelitis optica-like disease driven by a peptide of myelin oligodendroglial glycoprotein. Mult Scler 18:398-408

21. Vaknin-Dembinsky A, Brill L, Kassis I, Petrou P, Ovadia H, Ben-Hur T, Abramsky O, Karussis D (2012) T-cell reactivity against AQP4 in neuromyelitis optica. Neurology 79:945-946

22. Varrin-Doyer M, Spencer CM, Schulze-Topphoff U, Nelson PA, Stroud RM, Cree BA, Zamvil SS (2012) Aquaporin 4-specific T cells in neuromyelitis optica exhibit a Th17 bias and recognize Clostridium ABC transporter. Ann Neurol 72:53-64

23. Harrison LC (2008) Vaccination against self to prevent autoimmune disease: the type 1 diabetes model. Immunol Cell Biol 86:139-145

24. Pohl M, Fischer MT, Mader S, Schanda K, Kitic M, Sharma R, Wimmer I, Misu T, Fujihara K, Reindl M, Lassmann H, Bradl M (2011) Pathogenic T cell responses against aquaporin 4. Acta Neuropathol 122:21-34

\section{Submit your next manuscript to BioMed Central and take full advantage of:}

- Convenient online submission

- Thorough peer review

- No space constraints or color figure charges

- Immediate publication on acceptance

- Inclusion in PubMed, CAS, Scopus and Google Scholar

- Research which is freely available for redistribution 